\title{
Determination of bilateral symmetry of carotid artery structure and function in children and adolescents
}

This article was published in the following Dove Press journal:

Journal of Vascular Diagnostics and Interventions

13 January 2017

Number of times this article has been viewed

\author{
Katelyn E Uithoven' \\ Justin R Ryder ${ }^{2}$ \\ Roland Z Brown ${ }^{3}$ \\ Kyle D Rudser ${ }^{3}$ \\ Nicholas G Evanoff ${ }^{1}$ \\ Donald R Dengel ${ }^{1,2}$ \\ Aaron S Kelly ${ }^{2,4}$
}

'School of Kinesiology, University of Minnesota, ${ }^{2}$ Department of Pediatrics, University of Minnesota Medical School, ${ }^{3}$ Division of Biostatistics, School of Public Health, University of Minnesota, ${ }^{4}$ Department of Medicine, University of Minnesota Medical School, Minneapolis, MN, USA
Correspondence: Aaron S Kelly Departments of Pediatrics and Medicine, University of Minnesota Medical School, 420 Delaware Street S.E., MMC 7I5, Minneapolis, MN 55455, USA

Tel + I 6126263492

Email kelly105@umn.edu
Abstract: The carotid artery represents an ideal location for noninvasive assessment of subclinical atherosclerosis in youth. Examination of arterial structure and function is generally conducted in the left common carotid. However, if the left common carotid is inaccessible or provides a poor acoustic window, it is unknown if the right common carotid can provide comparable values. The symmetry of carotid arteries in youth with high-resolution ultrasound was compared. Participants ( $\mathrm{N}=230$ [121 females], 13.8 \pm 2.9 years old) were assessed for carotid intima media thickness (cIMT), carotid lumen diameter (cLD), carotid incremental elastic modulus (cIEM), carotid diameter compliance (cDC), carotid cross-sectional compliance (cCSC), carotid diameter distensibility (cDD), and carotid cross-sectional distensibility (cCSD). No significant differences $(P>0.05$ all $)$ were found for cIMT $(0.49 \pm 0.09$ vs $0.49 \pm 0.08 \mathrm{~mm})$, cIEM $(1095 \pm 382$ vs $1116 \pm 346 \mathrm{mmHg})$, cDC $(0.01 \pm 0.0$ vs $0.01 \pm 0.0 \mathrm{~mm} / \mathrm{mmHg})$, cCSC $(0.01 \pm 0.001 / \mathrm{mmHg}$ vs $0.01 \pm 0.001 / \mathrm{mmHg})$, cDD $(14.0 \% \pm 3.16 \%$ vs $13.7 \% \pm 3.18 \%)$, and $\mathrm{cCSD}(30.1 \% \pm 7.37 \%$ vs $29.4 \% \pm 7.36 \%)$. Significant differences were found for cLD $(6.06 \pm 0.62 \mathrm{~mm}$ vs $6.33 \pm 0.64 \mathrm{~mm}$, $P<0.001)$. The majority of measures for arterial structure and function are comparable between the left and right common carotid arteries. There were differences present for cLD; however, these discrepancies are likely due to anatomical differences between the left and right common carotid arteries. Therefore, if the left common carotid is unable to be assessed properly, the right common carotid may be a viable alternative measurement location of subclinical atherosclerosis. Keywords: vasculature, pediatric, ultrasound, carotid artery, intima media thickness

\section{Introduction}

Utilizing noninvasive techniques for the detection of atherosclerotic development and progression can improve risk stratification. ${ }^{1,2}$ This may be achieved through early identification of vascular adaptations known to be along the pathway of disease progression, which may indicate increased cardiovascular risk later in life. ${ }^{3-5}$ The carotid artery is commonly used for this purpose, as it is a large conduit vessel in an accessible location for ultrasound assessment ${ }^{6}$ and has been documented to have atherosclerotic lesion development in early life. ${ }^{7}$

The common carotid artery can be evaluated for both arterial structural and functional characteristics. ${ }^{8-10}$ Utilizing both sides of the common carotid artery as an average for measurement is a standard practice in pediatrics; ${ }^{2}$ however, when this cannot be done because of technical and/or anatomical issues, single carotid artery analysis is often necessary. It is important to be aware of any structural or functional differences between the left and right sides that may influence these values. Therefore, the question of whether bilateral symmetry exists between the left and right common 
carotid arteries is meaningful for these evaluations. In adult cadaver donors, carotid functional measures were similar even in the presence of anatomical differences. ${ }^{11}$ This study utilized harvested carotid trees, which may not elicit the same properties as intact vasculature in a growing body. Whether these carotid functional similarities apply to children and adolescents remains unknown.

\section{Objective}

This study sought to compare the structural and functional characteristics of the left and right common carotid arteries in children and adolescents with a wide range of body mass index (BMI) values and pubertal development stages.

\section{Materials and methods}

\section{Study design and participants}

Children and adolescents aged 8-18 years were included in this cross-sectional analysis. Participants were drawn from a large, ongoing study examining cardiovascular risk factors in youth ranging from normal weight to severe obesity. Participants with obesity or severe obesity were recruited from the University of Minnesota Masonic Children's Hospital Pediatric Weight Management Clinic and other participants were recruited from the local community as previously described. ${ }^{12}$ Individuals were excluded if they had cardiovascular disease or were taking medications known to influence cardiovascular function. The study protocol was approved by the University of Minnesota Institutional Review Board, and written informed consent/ assent was obtained from parents/participants.

\section{Anthropometrics and pubertal maturation}

All tests were performed in the morning after the participants had fasted (including no caffeine consumption) for a minimum of $12 \mathrm{~h}$. Height and weight were determined using a standard stadiometer (Model S100; Ayrton, Prior Lake, MN, USA) and an electronic scale (Serial No. 5002-8893; ST Scale-Tronix, White Plains, NY, USA), respectively. BMI was calculated as the body weight in kilograms divided by the height in meters squared. BMI percentiles were determined using age- and sex-based definitions from the Centers for Disease Control and Prevention. ${ }^{13}$ Normal weight was defined as $\geq 5$ th to $<85$ th percentile, overweight/obesity was defined as $\geq 85$ th to $<120 \%$ of the 95 th percentile, and severe obesity was defined as $\geq 120 \%$ of the 95 th percentile or an absolute BMI $\geq 35 \mathrm{~kg} / \mathrm{m}^{2}$ as previously described. ${ }^{14,15}$ Tanner (pubertal) stage was determined by a trained pediatrician or registered nurse.

\section{Blood pressure and heart rate}

Seated blood pressure (BP) and heart rate (HR) were measured after the participant had been resting quietly without legs crossed for 10 minutes. BP and HR were measured three consecutive times with an automated BP cuff (Model BP-8800 C, Colin Press-Mate; Colin Medical Instruments Corp., San Antonio, TX, USA) at $~ 3$ minute intervals following protocol derived from our previous study. ${ }^{12}$ The average of the three respective BP and HR measurements was used. Systolic BP (SBP) percentile was determined from age, sex, and height-specific values derived from the Fourth Report on the Diagnosis, Evaluation, and Treatment of High Blood Pressure in Children and Adolescents. ${ }^{16}$

\section{Assessments of carotid artery structure and function}

After participants rested in a supine position for 15 minutes in a quiet, climate-controlled room $\left(22^{\circ} \mathrm{C}-23^{\circ} \mathrm{C}\right)$ vascular images of both left and right common carotid arteries were obtained at end-diastole (gated by R wave on ECG) using B-mode ultrasound images (Acuson, Sequoia 512; Siemens Medical Solutions USA, Inc., Mountain View, CA, USA) of the far wall. An 8-15 MHz linear array transducer probe was held at constant distance from the skin at a fixed point over the cross-sectional axis of the imaged artery. The transducer remained at a fixed position over the common carotid artery $\sim 1-5 \mathrm{~cm}$ proximal from the carotid bifurcation capturing vascular parameters. Measurements for carotid intima media thickness (cIMT) were targeted at the distal $10 \mathrm{~mm}$ of the common carotid artery as recommended by pediatric guidelines. ${ }^{17}$ A standard electronic wall-tracking software program was used for the analysis of carotid structure and function (Vascular Research Tools 5; Medical Imaging Application LLC, Iowa City, IA, USA). Our laboratory has previously documented excellent cIMT reproducibility. ${ }^{18}$ Images were collected at 20 frames/s for $10 \mathrm{~s}$ (200 frames) for measurement of full arterial diameter change over the cardiac cycle to determine carotid lumen diameter (cLD), compliance, and distensibility, which were calculated based on mean diameter for the $10 \mathrm{~s}$ cycle.

\section{Carotid artery variable definitions and equations}

The following variable definitions and equations were utilized for comparison of left and right carotid artery properties: cIMT $(\mathrm{mm})$ defined as the delicate inner layer of the carotid artery wall; cLD ( $\mathrm{mm}$ ) defined as the measurement of the inner space (lumen) of the carotid artery calculated as the mean inner wall diameter minus cIMT in the longitudinal plane; carotid 
diameter compliance (cDC, $\mathrm{mm} / \mathrm{mmHg})$ calculated by ( $\left[D_{\max }-\right.$ $\left.D_{\text {min }}\right] / \Delta P$ ); carotid cross-sectional compliance (cCSC, $1 / \mathrm{mmHg}$ ) calculated by $\left(\left[\pi\left\{D_{\max } / 2\right\}^{2}-\pi\left\{D_{\min } / 2\right\}^{2}\right] /\left[\pi\left\{D_{\min } / 2\right\}^{2} \times \Delta P\right]\right)$; carotid incremental elastic modulus (cIEM, mmHg) calculated by $3\left(1+\left[\pi\left\{0.5 D_{\text {max }}\right\}^{2} / \pi\left\{0.5 D_{\text {min }}\right\}^{2}\right] / \mathrm{cCSC}\right)$; carotid diameter distensibility (cDD, \%) calculated by $\left(\left[D_{\max }-D_{\min }\right] / D_{\min }\right) \times$ $100 \%$; carotid cross-sectional distensibility (cCSD, \%) calculated by $\left(\left[\pi\left\{0.5 D_{\text {max }}\right\}^{2}-\pi\left\{0.5 D_{\text {min }}\right\}^{2}\right] / \pi\left[0.5 D_{\min }\right]^{2}\right) \times 100 \%$.

\section{Statistical analysis}

Descriptive statistics were tabulated for all participants and included mean and standard deviation for continuous variables and frequency, with percentage, for categorical variables. Left and right carotid artery outcome measurements were compared using Bland-Altman plots and paired $t$-tests. All analyses were conducted using R v3.1.1. ${ }^{19}$

\section{Results}

The mean age of the participants $(\mathrm{N}=230)$ was $13.8 \pm 2.9$ years, the mean BMI percentile was $84.2 \% \pm 24.2 \%$, the mean systolic BP percentile was $66.1 \% \pm 26.9 \%$, and the mean diastolic BP percentile was $37.7 \pm 22.4$ (Table 1). The sample was almost equally represented by males and females (105 [45.7\%] and 125 [54.3\%], respectively). There was roughly an even representation across Tanner stages: Stage 1=59 (25.0\%), Stage 2=52 (22.0\%), Stage 3=43 (18.2\%), Stage $4=53(22.5 \%)$, and Stage 5=29 (12.3\%). Participants were primarily White 189 (83\%).

No significant differences were found between left and right cIMT $(P=0.927)$, cIEM $(P=0.387), \operatorname{cDC}(P=0.137)$, $\operatorname{cCSC}(P=0.688), \operatorname{cDD}(P=0.203)$, or $\operatorname{cCSD}(P=0.159)$ (Table 2). Significant differences were found in cLD $(P<0.001)$ (Table 2).

Percent difference was calculated as (right - left)/left. Left and right common carotid arteries percent difference $>10 \%$ ranged from $32.2 \%$ to $62.2 \%,>20 \%$ ranged from $13.5 \%$ to
Table I Demographic characteristics of study participants

\begin{tabular}{ll}
\hline Study parameters & $\mathbf{N}=\mathbf{2 3 0}$ \\
\hline Age (years) & $13.8 \pm 2.9$ \\
Sex (\% female) & $125(54.3 \%)$ \\
Race (\% Caucasian) & $189(83.3 \%)$ \\
Tanner stage, $\mathrm{n}(\%)$ & \\
$\quad$ Stage I & $55(23.9)$ \\
Stage 2 & $5 \mathrm{I}(22.2)$ \\
Stage 3 & $4 \mathrm{I}(17.8)$ \\
Stage 4 & $51(22.2)$ \\
Stage 5 & $30(13.0)$ \\
Unreported & $2(0.9)$ \\
Body mass index (kg/m²) & $27.9 \pm 8.1$ \\
Body mass index percentile (\%) & $84.2 \pm 24.2$ \\
Systolic blood pressure (mmHg) & $116 \pm 13$ \\
Systolic blood pressure percentile (\%) & $66 \pm 27$ \\
Diastolic blood pressure (mmHg) & $59.3 \pm 8.39$ \\
Diastolic blood pressure percentile (\%) & $37.7 \pm 22.4$ \\
Heart rate (bpm) & $75 \pm 1 \mathrm{I}$ \\
\hline Note: Data repor
\end{tabular}

Note: Data reported as mean \pm standard deviation and frequency (\%).

$39.1 \%$, with cIEM displaying the greatest difference in both categories (Table 2). One measurement outlier was excluded from the data set that was unable to be reanalyzed.

\section{Discussion}

This is the first study, to our knowledge, to compare the anatomical and functional characteristics between the left and right common carotid arteries using noninvasive ultrasound in children and adolescents. On a cohort level, our observations suggest overall, albeit not perfect, symmetry of compliance, distensibility, and cIMT between the left and right common carotid arteries. We suggest that, in the research setting, values can be used interchangeably in cases where one vessel may be inaccessible or suffers from poor image quality. However, a fair amount of individual participant-level variability was observed, suggesting that values should not be used interchangeably in the clinical setting.

Table 2 Left and right carotid artery measurements

\begin{tabular}{|c|c|c|c|c|c|c|}
\hline \multirow[t]{2}{*}{ Covariate } & Left carotid & Right carotid & Difference $(95 \% \mathrm{Cl})$ & \multirow[t]{2}{*}{$P$-value } & \multirow{2}{*}{$\frac{>10 \% \text { diff }}{(\mathrm{N}=\mathbf{2 3 0})}$} & \multirow{2}{*}{$\frac{>20 \% \text { diff }}{(N=230)}$} \\
\hline & $(\mathbf{N}=\mathbf{2 3 0})$ & $(\mathrm{N}=\mathbf{2 3 0})$ & $(\mathbf{N}=\mathbf{2 3 0})$ & & & \\
\hline $\mathrm{cLD}(\mathrm{mm})$ & $6.08(0.56)$ & $6.27(0.58)$ & $-0.194(-0.253,-0.135)$ & $<0.001$ & 44 (19.1\%) & $3(1.3 \%)$ \\
\hline clMT (mm) & $0.49(0.09)$ & $0.49(0.08)$ & $0.000392(-0.00799,0.00878)$ & 0.927 & $74(32.2 \%)$ & $31(13.5 \%)$ \\
\hline cDD (\%) & $14.0(3.16)$ & $13.7(3.18)$ & $0.244(-0.133,0.621)$ & 0.203 & $127(55.2 \%)$ & $67(29.1 \%)$ \\
\hline cCSD (\%) & $30.1(7.37)$ & $29.4(7.36)$ & $0.628(-0.248,1.5)$ & 0.159 & $130(56.5 \%)$ & $73(31.7 \%)$ \\
\hline $\mathrm{cDC}(\mathrm{mm} / \mathrm{mmHg})$ & $0.01(0.0)$ & $0.01(0.0)$ & $-0.000334(-0.000775,0.000107)$ & 0.137 & 127 (55.2\%) & $60(26.1 \%)$ \\
\hline $\operatorname{cCSC}(\mathrm{I} / \mathrm{mmHg})$ & $0.01(0.0)$ & $0.01(0.0)$ & $-7.56 \mathrm{e}-05(-0.000447,0.000296)$ & 0.688 & $135(58.7 \%)$ & $77(33.5 \%)$ \\
\hline $\mathrm{clEM}(\mathrm{mmHg})$ & $1095(382.5)$ & $1116(346.5)$ & $-19.5(-63.9,24.8)$ & 0.387 & $143(62.2 \%)$ & $90(39.1 \%)$ \\
\hline
\end{tabular}

Notes: Values presented are mean (SD) or N (\%) where indicated. P-values calculated from paired tests. Percent difference was calculated as (right - left)/left.

Abbreviations: $\mathrm{CLD}$, carotid lumen diameter; cIMT, carotid intima media thickness; cDD, carotid diameter distensibility; cCSD, carotid cross-sectional distensibility; cDC, carotid diameter compliance; cCSC, carotid cross-sectional compliance; clEM, carotid incremental elastic modulus; Cl, confidence interval; diff, difference; SD, standard deviation. 
An anatomical difference in cLD between the left and right common carotid arteries in children and adolescents was observed. Another study, utilizing autopsy data in children and adolescents, reported similar findings. ${ }^{7}$ In contrast, several studies investigating this measurement in adults found no significant difference in cLD between both carotids, suggesting this anatomical distinction may diminish with maturity. ${ }^{20,21}$ As found in adult cadavers, ${ }^{11}$ no meaningful functional differences between the left and right carotid arties were observed even in the presence of anatomical differences. Blood volume, flow patterns, and shear stress in these areas are indicative of structural remodeling of the arterial vessel. ${ }^{22-24}$ One interesting finding in this study is that despite this anatomical difference in vessel diameter, compliance and distensibility characteristics were relatively similar between the left and right carotid arteries. Rodriquez et al performed a similar side-by-side comparison in carotid arteries of untreated hypertensive adults and found significantly higher cIMT values in the left common carotid, which they attributed to increased hemodynamic stress and intimal damage. ${ }^{20}$ This difference was not present in our sample of children and adolescents. The findings of Rodriquez et al could be explained by increased levels of perpendicular shear stress exerted on the carotid wall resulting from the cumulative exposure to hypertension over time. Because of this, the results of this study apply specifically to the pediatric population, primarily those absent of subclinical atherosclerosis development.

\section{Strengths and limitations}

The strengths of this study include a relatively large sample size representing a wide range of BMI values and an even distribution of sex and pubertal status. Limitations include the cross-sectional design of the study; therefore, whether the similarity of these carotid vascular beds remains stable over time cannot be determined. Because of the high percentage of Caucasians in this study, conclusions may not be applicable to all races. Furthermore, the data gathered in this study cannot conclusively determine whether the right common carotid measures are useful predictive measures compared to the left side due to study design, therefore further evaluation is required to investigate this.

\section{Conclusion}

In summary, results of this study indicate that, on a cohort basis, there is reasonable overall symmetry between the left and right common carotid arteries for noninvasive ultrasound measures of structure and function among healthy children and adolescents. Therefore, values could be used interchangeably in a healthy population for the research setting if technical limitations preclude the use of one side or the other. Although no significant differences were observed between the left and right common carotid arteries for vascular structure and function, a moderate degree of individual variability was noted for some measures of arterial stiffness. Although this study focused on the common carotid artery, future studies are encouraged to measure the internal carotid and bulb to further investigate potential differences in the pediatric population. Different carotid functional characteristics may be present with disease progression; therefore, further research should investigate left and right common carotid features in various disease states and pathological changes over time.

\section{Acknowledgments}

Research reported in this publication was supported by the National Heart, Lung, and Blood Institute of the National Institutes of Health under Award Number R01HL110957 (awarded to ASK), and an individual National Research Service Award from the National Heart, Lung, and Blood Institute (F32 HL127851-01 awarded to JRR). The content is solely the responsibility of the authors and does not necessarily represent the official views of the National Institutes of Health. Additional support was provided by the National Center for Advancing Translational Sciences (UL1TR000114).

The authors would like to thank the participants for donating their time to take part in this study. Also, the authors would like to thank Ms Annie Sheldon for coordinating the study and Ms Cameron Naughton for her program management expertise.

\section{Disclosure}

The authors report no conflicts of interest in this work.

\section{References}

1. Li S, Chen W, Srinivasan SR, et al. Childhood cardiovascular risk factors and carotid vascular changes in adulthood: the Bogalusa Heart Study. JAMA. 2003;290(17):2271-2276.

2. Urbina EM, Williams RV, Alpert BS, et al. Noninvasive assessment of subclinical atherosclerosis in children and adolescents: recommendations for standard assessment for clinical research: a scientific statement from the American Heart Association. Hypertension. 2009;54(5): 919-950.

3. Raitakari OT, Juonala M, Kahonen M, et al. Cardiovascular risk factors in childhood and carotid artery intima-media thickness in adulthood: the Cardiovascular Risk in Young Finns Study. JAMA. 2003; 290(17):2277-2283.

4. Tounian P, Aggoun Y, Dubern B, et al. Presence of increased stiffness of the common carotid artery and endothelial dysfunction in severely obese children: a prospective study. Lancet. 2001;358(9291):1400-1404.

5. Short KR, Blackett PR, Gardner AW, Copeland KC. Vascular health in children and adolescents: effects of obesity and diabetes. Vasc Health Risk Manage. 2009;5:973-990. 
6. Marlatt KL, Kelly AS, Steinberger J, Dengel DR. The influence of gender on carotid artery compliance and distensibility in children and adults. J Clin Ultrasound. 2013;41(6):340-346.

7. Solberg LA, Strong JP. Risk factors and atherosclerotic lesions. A review of autopsy studies. Arteriosclerosis. 1983;3(3):187-198.

8. Kassab GS, Molloi S. Cross-sectional area and volume compliance of porcine left coronary arteries. Am J Physiol. 2001;281(2):H623-H628.

9. Gamble G, Zorn J, Sanders G, MacMahon S, Sharpe N. Estimation of arterial stiffness, compliance, and distensibility from M-mode ultrasound measurements of the common carotid artery. Stroke. 1994;25(1): 11-16.

10. Skilton MR, Celermajer DS. Endothelial dysfunction and arterial abnormalities in childhood obesity. Int J Obes. 2006;30(7):1041-1049.

11. Adams GJ, Simoni DM, Bordelon CB, Jr, et al. Bilateral symmetry of human carotid artery atherosclerosis. Stroke. 2002;33(11):2575-2580.

12. Ryder JR, O'Connell M, Bosch TA, et al. Impaired cardiac autonomic nervous system function is associated with pediatric hypertension independent of adiposity. Pediatr Res. 2016;79(1-1):49-54.

13. Kuczmarski RJ, Ogden CL, Grummer-Strawn LM, et al. CDC growth charts: United States. Adv Data. 2000;(314):1-27.

14. Kelly AS, Barlow SE, Rao G, et al. Severe obesity in children and adolescents: identification, associated health risks, and treatment approaches: a scientific statement from the American Heart Association. Circulation. 2013;128(15):1689-1712.

15. Ryder JR, O'Connell MJ, Rudser KD, et al. Reproducibility of circulating endothelial cell enumeration and activation in children and adolescents. Biomark Med. 2016;10(5):463-471.
16. National High Blood Pressure Education Program Working Group on High Blood Pressure in Children and Adolescents. The fourth report on the diagnosis, evaluation, and treatment of high blood pressure in children and adolescents. Pediatrics. 2004;114(2 Suppl 4th Report):555-576.

17. Urbina EM, Williams RV, Alpert BS, et al. Noninvasive assessment of subclinical atherosclerosis in children and adolescents: recommendations for standard assessment for clinical research: a scientific statement from the American Heart Association. Hypertension. 2009;54(5):919-950.

18. Dengel DR, Jacobs DR, Steinberger J, Moran AM, Sinaiko AR. Gender differences in vascular function and insulin sensitivity in young adults. Clin Sci. 2011;120(4):153-160.

19. R: A language and environment for statistical computing. $R$ Foundation for Statistical Computing 2014. Available from: http://www.R-project. org/. Accessed March 30, 2016.

20. Rodriguez Hernandez SA, Kroon AA, van Boxtel MP, Mess WH, Lodder J, Jolles J, de Leeuw PW. Is there a side predilection for cerebrovascular disease? Hypertension. 2003;42(1):56-60.

21. Limbu YR, Gurung G, Malla R, Rajbhandari R, Regmi SR. Assessment of carotid artery dimensions by ultrasound in non-smoker healthy adults of both sexes. Nepal Med Coll J. 2006;8(3):200-203.

22. Korshunov VA, Berk BC. Flow-induced vascular remodeling in the mouse: a model for carotid intima-media thickening. Arterioscler Thromb Vasc Biol. 2003;23(12):2185-2191.

23. Ward MR, Pasterkamp G, Yeung AC, Borst C. Arterial remodeling. Mechanisms and clinical implications. Circulation. 2000;102(10):1186-1191.

24. Green DJ. Exercise training as vascular medicine: direct impacts on the vasculature in humans. Exerc Sport Sci Rev. 2009;37(4):196-202.
Journal of Vascular Diagnostics and Interventions

\section{Publish your work in this journal}

The Journal of Vascular Diagnostics and Interventions is an international, peer-reviewed journal of diagnostics, focusing on non invasive vascular investigation methods involved in the evaluation of vascular diseases. The journal is committed to the rapid publication in the fields of vascular diseases. Original research, review, case reports, expert

\section{Dovepress}

opinion and commentaries are all considered for publication. The manuscript management system is completely online and includes a very quick and fair peer-review system, which is all easy to use. Visit http://www.dovepress.com/testimonials.php to read real quotes from published authors. 